\title{
Elastomer Compatibility Testing of Renewable Diesel Fuels
}

Technical Report NREL/TP-540-38834

November 2005

\section{E. Frame}

Southwest Research Institute

R.L. McCormick

National Renewable Energy Laboratory

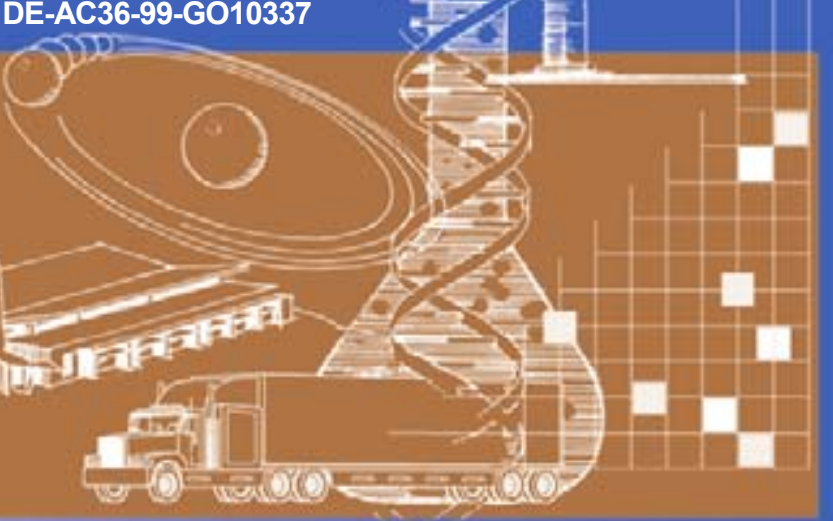




\section{Elastomer Compatibility Testing of Renewable Diesel Fuels}

E. Frame

Southwest Research Institute

R.L. McCormick

National Renewable Energy Laboratory

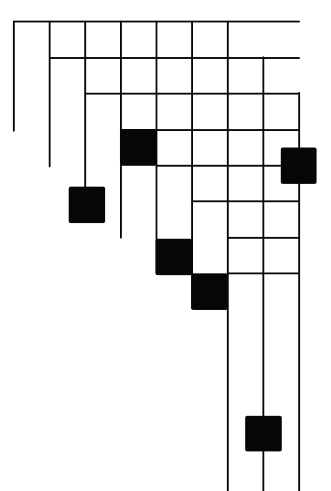

Prepared under Task No. FC06.9400

National Renewable Energy Laboratory

1617 Cole Boulevard, Golden, Colorado 80401-3393

303-275-3000 • www.nrel.gov

Operated for the U.S. Department of Energy

Office of Energy Efficiency and Renewable Energy

by Midwest Research Institute - Battelle

Contract No. DE-AC36-99-G010337 


\section{NOTICE}

This report was prepared as an account of work sponsored by an agency of the United States government. Neither the United States government nor any agency thereof, nor any of their employees, makes any warranty, express or implied, or assumes any legal liability or responsibility for the accuracy, completeness, or usefulness of any information, apparatus, product, or process disclosed, or represents that its use would not infringe privately owned rights. Reference herein to any specific commercial product, process, or service by trade name, trademark, manufacturer, or otherwise does not necessarily constitute or imply its endorsement, recommendation, or favoring by the United States government or any agency thereof. The views and opinions of authors expressed herein do not necessarily state or reflect those of the United States government or any agency thereof.

Available electronically at http://www.osti.gov/bridge

Available for a processing fee to U.S. Department of Energy and its contractors, in paper, from:

U.S. Department of Energy

Office of Scientific and Technical Information

P.O. Box 62

Oak Ridge, TN 37831-0062

phone: 865.576 .8401

fax: 865.576 .5728

email: mailto:reports@adonis.osti.gov

Available for sale to the public, in paper, from:

U.S. Department of Commerce

National Technical Information Service

5285 Port Royal Road

Springfield, VA 22161

phone: 800.553 .6847

fax: 703.605.6900

email: orders@ntis.fedworld.gov

online ordering: http://www.ntis.gov/ordering.htm 


\section{Table of Contents}

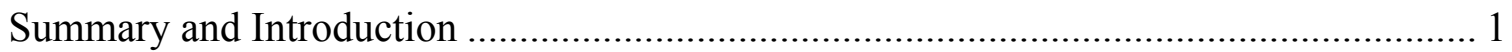

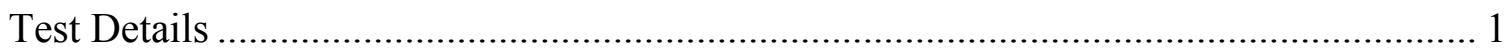

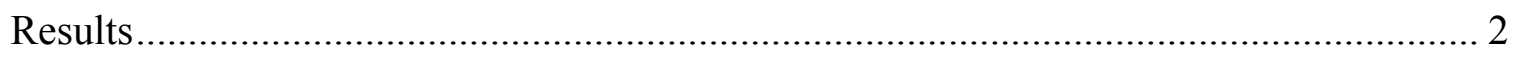

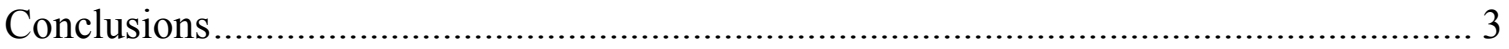

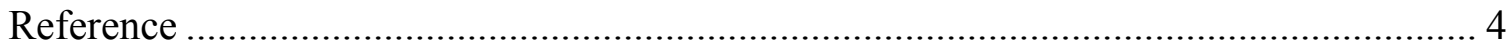

Appendix A: Certificate of Analysis—Diesel .05 LS Cert Fuel (\#2) ............................... 5

Appendix B: Raw Data, Data with Outliers Removed, Calculated Values, and Plots ....... 7

Appendix C: Bar Graphs with 95\% Confidence Bands............................................... 11 


\section{Summary and Introduction}

The U.S. Department of Energy (DOE) Fuels Technologies Program, under the DOE FreedomCAR and Vehicle Technologies Program, is charged with performing research to eliminate technical barriers to market penetration of renewable and synthetic fuels. One aspect of this program is to ensure that blends of petroleum-derived fuels and renewable fuels can be utilized without negative impact on engine durability. Materials compatibility is an important component of this research. The blending of renewable components, such as biodiesel or ethanol, into diesel fuel may impact the integrity and performance of various fuel system elastomers. The Southwest Research Institute conducted this work for the National Renewable Energy Laboratory (NREL) as part of a program to determine how ethanol and biodiesel affect these materials.

Six elastomers (N1059, N674, N0497, V747, and V884) commonly used in automotive applications were tested with the following fuels:

- Certification diesel fuel

- Certification diesel fuel blended with $15 \%$ ethanol

- Certification diesel fuel blended with $20 \%$ soy-derived biodiesel.

The elastomers were examined for thickness, diameter, and break load both before and after soaking in these fuels for 500 hours at $40^{\circ} \mathrm{C}$. Additionally, a control set was exposed to dry air only for the same length of time.

The results indicate that all of these elastomers appear to be fully compatible with $20 \%$ biodiesel blends. Overall, for each elastomer type, the ethanol-containing fuel had the largest impact on elastomer properties after storage. Thus these elastomers may not be fully compatible in all applications with fuels containing $15 \%$ ethanol.

\section{Test Details}

The following fuels were included in the investigation:

1. Fuel designated CL02-576, which was a certification diesel fuel with $29.2 \%$ vol aromatics and 346 ppm sulfur. Appendix A features a certificate of analysis for this fuel.

2. Fuel CL02-577 was a blend of $15 \%$ vol ethanol and $85 \%$ vol certification diesel fuel (E15). The fuel-grade ethanol was supplied by NREL.

3. Fuel CL02-578 was a blend of $20 \%$ vol soy-biodiesel (B20) and $80 \%$ vol certification diesel fuel. The B-100 used for the blend was Soy Gold (AL-25842).

4. No fuel; a set of samples were exposed to air only and served as a baseline.

Both the biodiesel and fuel-grade ethanol met applicable ASTM standards.

The test matrix of elastomers (all tested as O-rings) included:

- N674 general purpose nitrile rubber

- N0497 high aceto-nitrile content rubber for better fuel resistance 
- N1059 peroxide-cured nitrile rubber

- V747 flourocarbon filled with carbon black

- V884 flourocarbon without carbon black.

These elastomers were chosen because they were the same used in a prior study of various fuel oxygenates [1].

All elastomer tests were performed using four specimens, so any outliers could be removed based on statistics or engineering judgment. The elastomers stored in fuel were exposed for 500 hours at $40^{\circ} \mathrm{C}$. The baseline samples were only exposed to ambient laboratory room temperature. The following measurements were made after storage on all samples:

- O-ring thickness

- O-ring inside diameter

- Break load

- Sample volume and tensile strength, calculated from the primary measurements.

\section{Results}

Appendix B includes raw data, data with outliers removed, calculated values, and plots. No individual sample results were dropped based on statistical considerations; however, the following three tests were removed based on engineering judgment because their break loads were unusually low:

- Sample 1 of N1059 in B20 (CL02-578)

- Sample 4 of V747 in E15 (CL02-577)

- Sample 2 of V884 in B20 (CL02-578).

It is interesting to note that no outliers were observed or rejected for elastomers soaked in the baseline diesel fuel.

Bar graphs with $95 \%$ confidence bands are presented for the following parameters in Appendix C:

- Break load

- Break stress

- O-ring inside diameter

- O-ring thickness

- O-ring volume.

The following statistically significant differences were observed within each elastomer type.

\section{N1059 Peroxide-Cured Nitrile Rubber}

The average break load and corresponding break stress were lowest when exposed to the ethanol-containing fuel. Compared to the baseline, break load was reduced by $32 \%$. The results with baseline fuel, biodiesel, and air were not significantly different. 
O-ring volume was the lowest for the samples stored in air, as well as for the samples stored in the fuels. With this elastomer, each fuel type produced the same level of swell (approximately 18\% compared to the baseline).

\section{N674 General Purpose Nitrile Rubber}

The average break load and stress was the lowest for samples exposed to the ethanolcontaining fuel (37\% less than baseline). Similar break load and stress values were observed with the base fuel and biodiesel. Samples exposed to air had the highest break load and stress.

O-ring volume was lowest for samples exposed to air. Samples exposed to base fuel and biodiesel had the same level of increased volume (approximately 14\%-18\%), while the samples exposed to the ethanol-containing fuel had the largest volume increased compared to the baseline $(35 \%)$.

\section{N0497 High Aceto-Nitrile Content Rubber}

Trends similar to N674 were observed. Break load was 13\% lower for ethanol-exposed samples, and volume increase was $11 \%$ compared to baseline. The results with baseline fuel, biodiesel, and air were not significantly different.

\section{V747 Flourocarbon Filled with Carbon Black}

The average break load and stress were substantially lower for the samples exposed to ethanol (32\% compared to baseline). O-ring volume increase was highest with the ethanol-containing fuel (7\%). The results with base fuel, biodiesel, and air were the same statistically.

\section{V884 Flourocarbon without Carbon Black}

Trends similar to V747 were observed. Compared to baseline, break load was reduced by $28 \%$, and volume increase was $10 \%$ for the fuel containing ethanol. The results with baseline fuel, biodiesel, and air were not significantly different.

\section{Conclusions}

In general the samples exposed to only air exhibited higher break load than samples exposed to any of the fuels. Samples exposed to the ethanol-diesel blend exhibited a significant reduction in break load for all elastomers except N0497. Samples exposed to B20 did not exhibit significantly different break load from those exposed to the baseline fuel. These observations are mirrored in the calculated break stress. O-ring dimensions and volume typically increase for O-rings soaked in fuel relative to the air-exposed controls. The largest increase in dimensions and volume is observed for the ethanoldiesel blends. Elastomers exposed to B20 did not exhibit significantly different dimensions or volume from those exposed to the baseline diesel fuel.

The results indicate that all of these elastomers appear to be fully compatible with $20 \%$ biodiesel blends. Overall, for each elastomer type, the fuel containing ethanol had the 
largest impact on elastomer properties after storage. Thus these elastomers may not be fully compatible in all applications with fuels containing $15 \%$ ethanol.

\section{Reference}

1. Natarajan, M.; González, M.A.; Frame, E.A.; Naegeli, D.W.; Liney, E.; Asmus, T.; Piel, W.; Clark, W.; Wallace III, J.P; Garbak, J. "Oxygenates for Advanced PetroleumBased Diesel Fuels: Part 1. Screening and Selection Methodology for the Oxygenates" Society of Automotive Engineers Technical Paper No. 2001-01-3631; 2001. 


\section{Appendix A: \\ Certificate of Analysis-Diesel .05 LS Cert Fuel (\#2)}




\section{Chevron Phillips

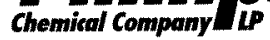

$5 m-4>/ 2-F$
DATE OF SHIPMENT

09-05-02

CUSTOMER PO NO.

282264-S

SALES ORDER NO. 5596161

TRAILER NO. 59-83007

MFG. DATE: 08-2002

\section{CERTIFICATE OF ANALYSIS}

DIESEL .05 LS CERT FUEL (\# 2) LOT 2HP05201

\section{TESTS}

Specific Gravity, 60/60 API Gravity

Corrosion, $50^{\circ} \mathrm{C}, 3 \mathrm{hrs}$

Sulfur, ppm

Flash Point, ${ }^{\circ} \mathrm{F}$

Pour Point, ${ }^{\circ} \mathrm{F}$

Cloud Point, ${ }^{\circ} \mathrm{F}$

Viscosity, $\operatorname{cs~} 40^{\circ} \mathrm{C}$

Carbon wt\%

Hydrogen wt $\%$

Carbon Density (gm/gal)

Net Heat of Combustion BTU/LB

Particulate Matter (mg/l)

Cetane Index

Cetane Number

\section{DISTILLATION, ${ }^{\circ} \mathrm{F}$}

IBP

$5 \%$

$10 \%$

$20 \%$

$30 \%$

$40 \%$

$50 \%$

$60 \%$

$70 \%$

$80 \%$

$90 \%$

$95 \%$

EP

Loss

Residue

\section{RESULTS}

0.8490
35.17
$1 A$
346
164.1
-10
+3
2.6
86.84
13.16
2785
18440
5.2
47.3
46.03

364.9

405.9

427.4

455.5

476.3

494.7

510.1

526.1

543.2

562.7

587.3

606.7

633.1

0.9

0.5

\section{SPECIFICATIONS METHOD}

$0.8398-0.8654$
$32-36$
$3 \mathrm{Max}$
$300-500$
$130 \mathrm{Min}$
$0 \mathrm{Max}$
Report
$2.2-3.2$
Report
Report
$2750-2806$
Report
$15 \mathrm{Max}$
$40-48$
$40-48$

$340-400$

$400-460$

$470-540$

$560-630$

$610-690$
ASTM D-4052

ASTM D-1298

ASTM D-130

ASTM D-2622

ASTM D-93

ASTM D-97

ASTM D-2500

ASTM D-445

Phillips

Phillips

Calculated

ASTM D-3338

ASTM D-2276

ASTM D-976

ASTM D-613

\section{ASTM D-86}

HYDROCARBON TYPE, VOL\%

Aromatics

Olefins

Saturates

\section{2 \\ 1.0}

69.8
$28-31$

Report

Report
D.g. Doerr teh

D.G. Doerr

Fuels Unit Team Leader 


\section{Appendix B: \\ Raw Data, Data with Outliers Removed, Calculated Values, and Plots}




\begin{tabular}{|c|c|c|c|c|c|c|c|c|c|c|c|c|c|c|c|}
\hline Elastomer & Fuel & Specimen ID & $\begin{array}{c}\text { Inside } \\
\text { Diameter } \\
\text { (in) }\end{array}$ & $\begin{array}{c}\text { Outside } \\
\text { Diameter } \\
\text { (in) }\end{array}$ & $\begin{array}{c}\text { O-ring } \\
\text { Diameter } \\
\text { (in) }\end{array}$ & $\begin{array}{l}\text { Break } \\
\text { Load } \\
\text { (lbs) }\end{array}$ & $\begin{array}{l}\text { Std dev } \\
\text { Break } \\
\text { Load } \\
\text { (lbs) }\end{array}$ & $\begin{array}{c}95 \% \text { C I } \\
\text { Break } \\
\text { Load } \\
\text { (lbs) }\end{array}$ & $\begin{array}{c}\text { Break } \\
\text { Stress } \\
(p s i)\end{array}$ & $\begin{array}{c}\text { Std dev } \\
\text { Break } \\
\text { Stress } \\
(p s i)\end{array}$ & $\begin{array}{c}95 \% \text { C I } \\
\text { Break } \\
\text { Stress } \\
\text { (psi) }\end{array}$ & $\begin{array}{c}\text { Average } \\
\text { Break } \\
\text { Stress } \\
\text { (psi) }\end{array}$ & $\begin{array}{l}\text { Volume } \\
\left(\text { (in^3) }^{\wedge}\right.\end{array}$ & $\begin{array}{c}\text { Average } \\
\text { Volume } \\
\text { (in^3) }\end{array}$ & $\begin{array}{l}95 \% \text { C.I. } \\
\text { Volume } \\
\text { (in^3) }\end{array}$ \\
\hline & & 1 & 1.034 & 1.182 & 0.0743 & 14.08 & & & 1626 & & & & \begin{tabular}{|l|}
0.015078 \\
\end{tabular} & 0.015169 & $\begin{array}{ll}6.65 \mathrm{E}-05 \\
\end{array}$ \\
\hline & I ${ }_{1} 02576$ & 2 & 1.033 & 1.182 & 0.0746 & 16.83 & & & 1927 & & & 1803 & \begin{tabular}{|l|}
0.015199 \\
\end{tabular} & & \\
\hline & CLO2-5/6 & 3 & 1.031 & 1.180 & 0.0745 & 16.58 & 1.26 & 1.24 & 1904 & 140 & 138 & 1803 & \begin{tabular}{|l|}
0.015135 \\
\end{tabular} & & \\
\hline & & 4 & 1.036 & 1.185 & 0.0746 & 15.36 & & & 1756 & & & & \begin{tabular}{|l|}
0.015262 \\
\end{tabular} & & \\
\hline & & 1 & 1.072 & 1.226 & 0.0774 & 12.55 & & & 1336 & & & & \begin{tabular}{|l|}
0.016967 \\
\end{tabular} & 0.017373 & 0.00026 \\
\hline & & 2 & 1.075 & 1.231 & 0.0782 & 11.83 & & & 1231 & & & & \begin{tabular}{|l|}
0.017415 \\
\end{tabular} & & \\
\hline $\mathrm{N} 6 / 4$ & CLO2-5// & 3 & 1.068 & 1.227 & 0.0791 & 13.30 & 1.14 & 1.11 & 1353 & 116 & 113 & 1255 & \begin{tabular}{|l|}
0.017726 \\
\end{tabular} & & \\
\hline & & 4 & 1.068 & 1.225 & 0.0784 & 10.63 & & & 1102 & & & & \begin{tabular}{|l|}
0.017384 \\
\end{tabular} & & \\
\hline & & 1 & 1.029 & 1.177 & 0.0740 & 15.36 & & & 1785 & & & & \begin{tabular}{|l|}
0.014916 \\
\end{tabular} & 0.014671 & $\begin{array}{l}000294 \\
\end{array}$ \\
\hline & & 2 & 1.029 & 1.176 & 0.0734 & 15.37 & & & 1816 & & & & \begin{tabular}{|l|}
0.014666 \\
\end{tabular} & & \\
\hline & CL02-578 & 3 & 1.031 & 1.174 & 0.0722 & 14.66 & 3.07 & 3.01 & 1792 & 28 & 28 & 1811 & \begin{tabular}{|l|}
0.014175 \\
\end{tabular} & & \\
\hline & & 4 & 1.029 & 1.177 & 0.0740 & 15.91 & & & 1848 & & & & \begin{tabular}{|l|}
0.014926 \\
\end{tabular} & & \\
\hline & & $\overline{11}$ & 1.185 & \begin{tabular}{c|}
1.324 \\
\end{tabular} & 0.0694 & $\begin{array}{l}12.48 \\
\end{array}$ & & & 1648 & & & & \begin{tabular}{|l|}
0.014928 \\
\end{tabular} & 0.015211 & $\begin{array}{l}0.000159 \\
\end{array}$ \\
\hline & & 2 & 1.180 & 1.321 & 0.0705 & 12.26 & & & 1572 & & & & \begin{tabular}{|l|}
0.015328 \\
\end{tabular} & & \\
\hline & CL02-576 & 3 & 1.182 & 1.323 & 0.0703 & 12.22 & 0.12 & 0.12 & 1575 & 35 & 34 & 1598 & \begin{tabular}{|l|}
0.015269 \\
\end{tabular} & & \\
\hline & & 4 & 1.188 & 1.329 & 0.0702 & 12.38 & & & 1598 & & & & \begin{tabular}{|l|}
0.015317 \\
\end{tabular} & & \\
\hline & & 1 & 1.225 & 1.372 & 0.0734 & 11.28 & & & 1335 & & & & \begin{tabular}{|l|}
0.017248 \\
\end{tabular} & 0.016466 & 0.000935 \\
\hline & & 2 & 1.225 & 1.372 & 0.0735 & 11.39 & & & 1343 & & & 1302 & \begin{tabular}{|l|}
0.017311 \\
\end{tabular} & & \\
\hline N0497 & CL02-577 & 3 & 1.230 & 1.366 & 0.0682 & 11.01 & 0.16 & 0.16 & 1507 & 436 & 427 & 1392 & 0.014905 & & \\
\hline & & 4 & 1.219 & 1.363 & 0.0718 & 11.19 & & & 1384 & & & & \begin{tabular}{|l|}
0.016403 \\
\end{tabular} & & \\
\hline & & 1 & 1.185 & 1.323 & 0.0694 & 11.80 & & & 1559 & & & & \begin{tabular}{|l|}
0.014918 \\
\end{tabular} & 0.015207 & 0.000226 \\
\hline & & 2 & 1.189 & 1.330 & 0.0708 & 12.00 & & & 1527 & & & & \begin{tabular}{|l|}
0.015558 \\
\end{tabular} & & \\
\hline & CL02-578 & 3 & 1.173 & 1.314 & 0.0705 & 12.49 & 0.31 & 0.30 & 1601 & 34 & 33 & $15 / 0$ & \begin{tabular}{|l|}
0.015247 \\
\end{tabular} & & \\
\hline & & 4 & 1.175 & 1.315 & 0.0701 & 12.29 & & & 1592 & & & & \begin{tabular}{|l|}
0.015104 \\
\end{tabular} & & \\
\hline & & $\overline{11}$ & 1.243 & 1.386 & 0.0713 & 16.59 & & & 2078 & & & & \begin{tabular}{|l|}
0.016491 \\
\end{tabular} & 0.016964 & 0.000456 \\
\hline & lCI 02576 & 2 & 1.238 & 1.383 & 0.0730 & 17.36 & & & 2074 & & & 1948 & \begin{tabular}{|l|}
0.017239 \\
\end{tabular} & & \\
\hline & CLUL-5/ & 3 & 1.242 & 1.385 & 0.0714 & 17.66 & 2.56 & 2.51 & 2206 & 347 & 341 & 1948 & \begin{tabular}{|l|}
0.016527 \\
\end{tabular} & & \\
\hline & & 4 & 1.248 & 1.395 & 0.0735 & 12.16 & & & 1435 & & & & \begin{tabular}{|l|}
0.017599 \\
\end{tabular} & & \\
\hline & & 1 & 1.282 & 1.429 & 0.0733 & 9.34 & & & 1106 & & & & \begin{tabular}{|l|}
0.017993 \\
\end{tabular} & 0.01733 & 0.000521 \\
\hline N1050 & I & 2 & 1.283 & 1.423 & 0.0703 & 12.79 & & & 1649 & & & 1121 & \begin{tabular}{|l|}
0.016494 \\
\end{tabular} & & \\
\hline NTus9 & CLU $-5 / 1$ & 3 & 1.276 & 1.421 & 0.0726 & 12.10 & 1.52 & 1.49 & 1464 & 227 & 223 & 1421 & \begin{tabular}{|l|}
0.017509 \\
\end{tabular} & & \\
\hline & & 4 & 1.275 & 1.419 & 0.0722 & 11.99 & & & 1465 & & & & \begin{tabular}{|l|}
0.017325 \\
\end{tabular} & & \\
\hline & & 1 & 1.232 & 1.369 & 0.0688 & 6.97 & & & 939 & & & & \begin{tabular}{|l|}
0.015172 \\
\end{tabular} & 0.016084 & 0.000575 \\
\hline & lc1 02578 & 2 & 1.242 & 1.382 & 0.0702 & 16.84 & & & 2179 & & & 1798 & \begin{tabular}{|l|}
0.015934 \\
\end{tabular} & & \\
\hline & CLOZ-5/8 & 3 & 1.241 & 1.384 & 0.0715 & 18.15 & 5.00 & 4.90 & 2260 & 605 & 593 & $1 / 98$ & \begin{tabular}{|l|}
0.016567 \\
\end{tabular} & & \\
\hline & & 4 & 1.238 & 1.382 & 0.0718 & 14.69 & & & 1815 & & & & \begin{tabular}{|l|}
0.016664 \\
\end{tabular} & & \\
\hline & & 1 & $\begin{array}{l}0.863 \\
\end{array}$ & 1.001 & 0.0693 & $\begin{array}{l}13.91 \\
\end{array}$ & & & 1845 & & & & \begin{tabular}{|l|}
0.011044 \\
\end{tabular} & 0.011109 & 0.000441 \\
\hline & & 2 & 0.874 & 1.008 & 0.0669 & 13.73 & & & 1953 & & & & \begin{tabular}{|l|}
0.010397 \\
\end{tabular} & & \\
\hline & CL02-576 & 3 & 0.868 & 1.008 & 0.0702 & 14.51 & 0.36 & 0.35 & 1877 & 183 & 179 & 1854 & \begin{tabular}{|l|}
0.011394 \\
\end{tabular} & & \\
\hline & & 4 & 0.863 & 1.005 & 0.0710 & 13.77 & & & 1742 & & & & \begin{tabular}{|l|}
0.011603 \\
\end{tabular} & & \\
\hline & & 1 & 0.898 & 1.041 & 0.0718 & 9.88 & & & 1222 & & & & \begin{tabular}{|l|}
0.012321 \\
\end{tabular} & 0.011957 & 0.000233 \\
\hline & & 2 & 0.893 & 1.033 & 0.0700 & 9.41 & & & 1223 & & & 099 & \begin{tabular}{|l|}
0.011643 \\
\end{tabular} & & \\
\hline V747 & CL02-577 & 3 & 0.900 & 1.041 & 0.0707 & 9.66 & 3.59 & 3.51 & 1232 & 453 & 444 & 999 & \begin{tabular}{|l|}
0.011955 \\
\end{tabular} & & \\
\hline & & 4 & 0.900 & 1.041 & 0.0705 & 2.49 & & & 319 & & & & \begin{tabular}{|l|}
0.011909 \\
\end{tabular} & & \\
\hline & & 1 & 0.861 & 1.007 & 0.0731 & 15.02 & & & 1789 & & & & \begin{tabular}{|l|}
0.012324 \\
\end{tabular} & 0.01169 & 0.00065 \\
\hline & & 2 & 0.854 & 1.001 & 0.0736 & 14.32 & & & 1685 & & & & \begin{tabular}{|l|}
0.012393 \\
\end{tabular} & & \\
\hline & CL02-578 & 3 & 0.875 & 1.011 & 0.0684 & 13.76 & 0.56 & 0.55 & 1871 & 83 & 81 & 1798 & \begin{tabular}{|l|}
0.010901 \\
\end{tabular} & & \\
\hline & & 4 & 0.871 & 1.010 & 0.0693 & 13.93 & & & 1848 & & & & \begin{tabular}{|l|}
0.011141 \\
\end{tabular} & & \\
\hline & & $\overline{11}$ & (0.986 & \begin{tabular}{c|}
1.124 \\
\end{tabular} & 0.0688 & $=13.21$ & & & 1778 & & & & \begin{tabular}{|l|}
0.012319 \\
\end{tabular} & 0.012571 & 0.000182 \\
\hline & & 2 & 0.981 & 1.120 & 0.0695 & 13.00 & & & 1712 & & & 1749 & \begin{tabular}{|l|}
0.012536 \\
\end{tabular} & & \\
\hline & CL02-576 & 3 & 0.992 & 1.130 & 0.0693 & 13.46 & 0.19 & 0.18 & 1784 & 37 & 36 & 1749 & \begin{tabular}{|l|}
0.012579 \\
\end{tabular} & & \\
\hline & & 4 & 0.993 & 1.133 & 0.0700 & 13.25 & & & 1723 & & & & \begin{tabular}{|l|}
0.01285 \\
\end{tabular} & & \\
\hline & & 1 & 1.008 & 1.151 & 0.0719 & 10.28 & & & 1266 & & & & \begin{tabular}{|l|}
0.013783 \\
\end{tabular} & 0.013951 & 0.00052 \\
\hline V884 & & 2 & 1.013 & 1.154 & 0.0709 & 9.93 & & & 1259 & & & & \begin{tabular}{|l|}
0.013433 \\
\end{tabular} & & \\
\hline V884 & CL02-577 & 3 & 1.015 & 1.164 & 0.0743 & 10.10 & 0.37 & 0.36 & 1164 & 54 & 53 & 1215 & \begin{tabular}{|l|}
0.014856 \\
\end{tabular} & & \\
\hline & & 4 & 1.015 & 1.158 & 0.0716 & 9.43 & & & 1173 & & & & \begin{tabular}{|l|}
0.013733 \\
\end{tabular} & & \\
\hline & & 1 & 0.991 & 1.125 & 0.0668 & 12.82 & & & 1833 & & & & \begin{tabular}{|l|}
0.011634 \\
\end{tabular} & 0.012267 & 0.00051 \\
\hline & & 2 & 0.987 & 1.127 & 0.0698 & 6.30 & & & 823 & & & & \begin{tabular}{|l|}
0.012721 \\
\end{tabular} & & \\
\hline & CL02-578 & 3 & 0.988 & 1.123 & 0.0675 & 13.21 & 3.43 & 3.36 & 1848 & 494 & 484 & 1559 & \begin{tabular}{|l|}
0.01186 \\
\end{tabular} & & \\
\hline & & 4 & 0.988 & 1.128 & 0.0702 & 13.40 & & & 1733 & & & & \begin{tabular}{|l|}
0.012855 \\
\end{tabular} & & \\
\hline & & & & & & & & & & & & & & & \\
\hline & Air & 1 & 0.990 & 1.132 & 0.0708 & 19.4 & 0.11 & 0.11 & 2463 & 43 & 42.00 & & \begin{tabular}{|l|}
0.013114 \\
\end{tabular} & 0.012905 & 0.000151 \\
\hline$N G 47$ & & 2 & 0.989 & 1.130 & 0.0704 & 19.2 & & & 2465 & & & 2495 & \begin{tabular}{|l|}
0.01295 \\
\end{tabular} & & \\
\hline N64l & & 3 & 0.991 & 1.130 & 0.0696 & 19.4 & & & 2555 & & & 2495 & \begin{tabular}{|l|}
0.012678 \\
\end{tabular} & & \\
\hline & & 4 & 0.990 & 1.130 & 0.0702 & 19.3 & & & 2496 & & & & \begin{tabular}{|l|}
0.012877 \\
\end{tabular} & & \\
\hline & Air & 1 & 1.174 & 1.313 & 0.0695 & 12.9 & 0.54 & 0.53 & 1705 & 75 & 73.58 & & \begin{tabular}{|l|}
0.014821 \\
\end{tabular} & 0.014885 & $\begin{array}{l}.98 \mathrm{E}-05 \\
\end{array}$ \\
\hline A०107 & & 2 & 1.169 & 1.308 & 0.0698 & 12.6 & & & 1651 & & & 160 & \begin{tabular}{|l|}
0.014877 \\
\end{tabular} & & \\
\hline No49r & & 3 & 1.167 & 1.307 & 0.0699 & 12.5 & & & 1630 & & & 1696 & \begin{tabular}{|l|}
0.014926 \\
\end{tabular} & & \\
\hline & & 4 & 1.175 & 1.315 & 0.0697 & 13.7 & & & 1799 & & & & \begin{tabular}{|l|}
0.014916 \\
\end{tabular} & & \\
\hline & Air & 1 & 1.176 & 1.311 & 0.0677 & 19.5 & 2.05 & 2.01 & 2710 & 374 & 366.57 & & \begin{tabular}{|l|}
0.014062 \\
\end{tabular} & 0.014629 & 0.000686 \\
\hline N1050 & & 2 & 1.168 & 1.303 & 0.0673 & 17.4 & & & 2444 & & & 2076 & \begin{tabular}{|l|}
0.013805 \\
\end{tabular} & & \\
\hline N1059 & & 3 & 1.171 & 1.312 & 0.0703 & 14.5 & & & 1872 & & & $22 / 6$ & \begin{tabular}{|l|}
0.015156 \\
\end{tabular} & & \\
\hline & & 4 & 1.168 & 1.310 & 0.0712 & 16.5 & & & 2077 & & & & \begin{tabular}{|l|}
0.015492 \\
\end{tabular} & & \\
\hline & Air & $\overline{11}$ & 0.865 & 1.005 & 0.0701 & 15.2 & 0.49 & 0.48 & 1968 & 77 & 75.75 & & \begin{tabular}{|l|}
0.011328 \\
\end{tabular} & 0.011242 & $\begin{array}{l}0.000124 \\
\end{array}$ \\
\hline & & 2 & 0.866 & 1.004 & 0.0692 & 14.7 & & & 1953 & & & & \begin{tabular}{|l|}
0.011052 \\
\end{tabular} & & \\
\hline V747 & & 3 & 0.864 & 1.004 & 0.0697 & 14.4 & & & 1886 & & & 1901 & \begin{tabular}{|l|}
0.011202 \\
\end{tabular} & & \\
\hline & & 4 & 0.860 & 1.001 & 0.0704 & 14.0 & & & 1799 & & & & \begin{tabular}{|l|}
0.011386 \\
\end{tabular} & & \\
\hline & Air & 1 & 0.988 & 1.126 & 0.0687 & 14.4 & 0.65 & $\overline{0.64}$ & 1943 & 119 & 116.47 & & \begin{tabular}{|l|}
0.012325 \\
\end{tabular} & 0.012693 & 0.000238 \\
\hline V884 & & 2 & 0.989 & 1.130 & 0.0704 & 13.0 & & & 1676 & & & 1818 & \begin{tabular}{|l|}
0.012944 \\
\end{tabular} & & \\
\hline V884 & & 3 & 0.985 & 1.124 & 0.0696 & 14.3 & & & 1884 & & & 1818 & \begin{tabular}{|l|}
0.012615 \\
\end{tabular} & & \\
\hline & & 4 & 0.992 & 1.132 & 0.0701 & 13.7 & & & 1768 & & & & \begin{tabular}{|l|}
0.012888 \\
\end{tabular} & & \\
\hline
\end{tabular}




\begin{tabular}{|c|c|c|c|c|c|c|c|c|c|c|c|c|c|c|c|c|c|c|c|c|}
\hline Elastome & Fuel & Specimen ID & $\begin{array}{c}\begin{array}{c}\text { Inside } \\
\text { Diameter } \\
\text { (in) }\end{array} \\
\end{array}$ & $\begin{array}{c}\text { Outside } \\
\text { Diameter } \\
\text { (in) }\end{array}$ & $\begin{array}{c}\text { O-ring } \\
\text { Diameter } \\
\text { (in) }\end{array}$ & $\begin{array}{l}\text { Break } \\
\text { Load } \\
\text { (lbs) }\end{array}$ & $\begin{array}{l}\text { Std dev } \\
\text { Break } \\
\text { Load } \\
\text { (lbs) } \\
\end{array}$ & $\begin{array}{l}95 \% \mathrm{Cl} \\
\text { Break } \\
\text { Load } \\
\text { (lbs) }\end{array}$ & $\begin{array}{c}\text { Break } \\
\text { Stress } \\
(p s i)\end{array}$ & $\begin{array}{l}\text { Std dev } \\
\text { Break } \\
\text { Stress } \\
\text { (psi) }\end{array}$ & $\begin{array}{l}95 \% \mathrm{Cl} \\
\text { Break } \\
\text { Stress } \\
(\text { psi) }\end{array}$ & $\begin{array}{l}\text { Average } \\
\text { Break } \\
\text { Stress } \\
(p s i)\end{array}$ & $\begin{array}{c}\text { Break } \\
\text { Stress } \\
T\end{array}$ & $\begin{array}{l}\text { Volume } \\
\left(i n^{\wedge} 3\right)\end{array}$ & $\begin{array}{l}\text { Average } \\
\text { Volume } \\
\left.\text { (in } 3{ }^{\wedge} 3\right)\end{array}$ & $\begin{array}{l}95 \% \text { C.I. } \\
\text { Volume } \\
\left(i^{\wedge} \wedge 3\right)\end{array}$ & $\begin{array}{l}\text { avg } \\
\text { id } \\
\text { in }\end{array}$ & $\begin{array}{c}95 \% \mathrm{Cl} \\
\text { id } \\
\text { in }\end{array}$ & $\begin{array}{l}\text { avg } \\
\text { thickness } \\
\text { in }\end{array}$ & $\begin{array}{c}95 \% \mathrm{Cl} \\
\text { thickness } \\
\text { in }\end{array}$ \\
\hline \multirow[t]{13}{*}{ N674 } & CL02-576 & $\frac{1}{2}$ & $\begin{array}{l}1.034 \\
1033 \\
\end{array}$ & $\begin{array}{l}1.182 \\
1182 \\
\end{array}$ & \begin{tabular}{|l|}
0.0743 \\
0076 \\
\end{tabular} & $\begin{array}{l}14.08 \\
16.83\end{array}$ & & & $\begin{array}{l}1626 \\
1927 \\
\end{array}$ & & & & $\begin{array}{l}-1.26 \\
088\end{array}$ & \begin{tabular}{|l|}
0.015078 \\
0015199 \\
\end{tabular} & & & & & & \\
\hline & & $\frac{2}{3}$ & $\begin{array}{l}1.035 \\
1.031 \\
\end{array}$ & $\frac{1.102}{1.180}$ & \begin{tabular}{|l|}
0.0746 \\
0.0745 \\
\end{tabular} & $\begin{array}{l}10.03 \\
16.58 \\
\end{array}$ & 1.26 & 1.24 & $\begin{array}{l}1927 \\
1904 \\
\end{array}$ & 140 & 138 & 1803 & $\begin{array}{l}0.88 \\
0.72 \\
\end{array}$ & \begin{tabular}{|l|}
0.015135 \\
0.015135 \\
\end{tabular} & 0.015169 & \begin{tabular}{|l}
$7.80268 \mathrm{E}-05$ \\
\end{tabular} & 1.033 & 0.001661 & 0.074 & 0.000164 \\
\hline & & 1 & 1.036 & 1.185 & 0.0746 & $\begin{array}{l}15.36 \\
1255 \\
\end{array}$ & & & 1756 & & 138 & & -0.34 & \begin{tabular}{|l|l|}
0.015262 \\
011067
\end{tabular} & & & & & & \\
\hline & CLO02-577 & & $\frac{1.072}{1.075}$ & $\frac{1.226}{1.231}$ & \begin{tabular}{l|l}
0.0774 \\
0.0782
\end{tabular} & $\begin{array}{l}12.55 \\
11.83 \\
\end{array}$ & & & $\frac{1336}{1231}$ & & & & & \begin{tabular}{|l|}
0.016967 \\
0.017415 \\
\end{tabular} & & & & & & \\
\hline & & 3 & 1.068 & 1.227 & 0.0791 & 13.30 & 1.14 & 1.11 & 1353 & 116 & 113 & 1255 & & \begin{tabular}{|l|}
0.017726 \\
\end{tabular} & 0.017373 & \begin{tabular}{|l|}
0.000305667 \\
\end{tabular} & 1.071 & 0.003092 & 0.078 & 0.000709 \\
\hline & & 4 & 1.068 & 1.225 & 0.0784 & 10.63 & & & 1102 & & & & -1.33 & \begin{tabular}{|l|l|}
0.017384 \\
\end{tabular} & & & & & & \\
\hline & CL02-578 & & 1.029 & 1.177 & 0.0740 & 15.36 & & & 1785 & & & & & \begin{tabular}{|l|}
0.014916 \\
\end{tabular} & & & & & & \\
\hline & & $\frac{2}{2}$ & 1.029 & 1.176 & $\begin{array}{ll}0.0734 \\
0.0702\end{array}$ & 15.37 & & & 1816 & & & & & \begin{tabular}{|l|l|}
0.014666 \\
\end{tabular} & & & & & & \\
\hline & & $\frac{3}{4}$ & \begin{tabular}{|l|}
.031 \\
1029 \\
1029
\end{tabular} & $\begin{array}{l}1.174 \\
1177 \\
\end{array}$ & \begin{tabular}{|l|}
0.0722 \\
00740
\end{tabular} & $\begin{array}{l}14.66 \\
15.91\end{array}$ & 2.58 & 2.52 & $\begin{array}{l}1792 \\
1848\end{array}$ & 28 & 28 & 1811 & & \begin{tabular}{|l|l|l|l|l|}
0.0145 \\
\end{tabular} & \begin{tabular}{|l|l|}
0.014671 \\
\end{tabular} & 0.000344597 & 1.030 & 0.000702 & 0.073 & 0.00086 \\
\hline & Air & $\frac{4}{1}$ & $\begin{array}{l}1.029 \\
0.990\end{array}$ & $\begin{array}{l}1.177 \\
1.132\end{array}$ & \begin{tabular}{|l|}
0.00740 \\
0.0708 \\
\end{tabular} & $\begin{array}{l}\frac{15.91}{19.4} \\
\end{array}$ & & & $\begin{array}{l}1848 \\
2463\end{array}$ & & & & & $\left|\begin{array}{l}0.014926 \\
0.013114\end{array}\right|$ & & & & & & \\
\hline & & 2 & 0.989 & 1.130 & \begin{tabular}{|l|}
0.0704 \\
\end{tabular} & 19.2 & & & 2465 & & & & & \begin{tabular}{|l|}
0.01295 \\
0.01295 \\
\end{tabular} & & & & & & \\
\hline & & 3 & 0.991 & 1.130 & $\begin{array}{ll}0.0696 \\
0.0702\end{array}$ & 19.4 & 0.11 & 0.11 & 2555 & 43 & 42.00 & 2495 & & \begin{tabular}{|l|l|}
0.012678 \\
\end{tabular} & \begin{tabular}{|l|}
0.012905 \\
\end{tabular} & \begin{tabular}{|l|}
0.000177505 \\
\end{tabular} & 0.990 & 0.000775 & 0.070 & 0.000476 \\
\hline & & & & & & & & & & & & & & \begin{tabular}{|l|l|l|l|l|l|}
0.012877 \\
001928
\end{tabular} & & & & & & \\
\hline \multirow[t]{13}{*}{$\begin{array}{l}\text { N0497 } \\
\end{array}$} & CL02-576 & $\frac{1}{2}$ & $\begin{array}{l}1.185 \\
1.80 \\
\end{array}$ & $\begin{array}{l}1.324 \\
1321\end{array}$ & $\begin{array}{ll}0.0694 \\
00705\end{array}$ & $\begin{array}{l}12.48 \\
1.26\end{array}$ & & & $\begin{array}{l}1648 \\
1572\end{array}$ & & & & & \begin{tabular}{|l|}
0.014928 \\
001528 \\
\end{tabular} & & & & & & \\
\hline & & $\frac{2}{3}$ & $\begin{array}{l}1.180 \\
1.182 \\
\end{array}$ & \begin{tabular}{|l|l|}
1.321 \\
1.323 \\
\end{tabular} & \begin{tabular}{|l|l|}
0.0705 \\
0.0703
\end{tabular} & $\begin{array}{l}12.26 \\
12.22 \\
\end{array}$ & 0.12 & 0.12 & \begin{tabular}{|l|}
1572 \\
1575 \\
\end{tabular} & 35 & 34 & 1598 & & \begin{tabular}{|l|}
0.015328 \\
0.015269 \\
\end{tabular} & 0.015211 & \begin{tabular}{|l|}
0.000186108 \\
\end{tabular} & & 0.003321 & 0.070 & 0.000445 \\
\hline & & 4 & 1.188 & 1.329 & 0.0702 & 12.38 & & & 1598 & & & & & \begin{tabular}{|l|}
0.015317 \\
\end{tabular} & & 0.000180108 & 1.184 & 0.003321 & 0.010 & 0.000445 \\
\hline & CL02-577 & 1 & 1.225 & 1.372 & 0.0734 & 11.28 & & & 1335 & & & & & \begin{tabular}{|l|l|}
0.017248 \\
\end{tabular} & & & & & & \\
\hline & & & $\frac{1.225}{1.230}$ & $\begin{array}{l}1.372 \\
1365\end{array}$ & 0.0735 & $\begin{array}{l}11.39 \\
1101\end{array}$ & & & $\begin{array}{l}1343 \\
1507\end{array}$ & & & & & \begin{tabular}{|l|l|}
0.017311 \\
001905 \\
\end{tabular} & & & & & & \\
\hline & & $\frac{3}{4}$ & $\begin{array}{l}1.230 \\
1.219\end{array}$ & $\frac{1.366}{1.363}$ & \begin{tabular}{l|l}
0.0682 \\
0.0718
\end{tabular} & $\frac{11.01}{11.19}$ & 0.16 & 0.16 & $\begin{array}{l}1507 \\
1384\end{array}$ & 115 & 113 & 1392 & 1.00 & \begin{tabular}{|l|}
0.014905 \\
0.016403 \\
\end{tabular} & $0.016466 \mid$ & \begin{tabular}{|l|}
0.001097997 \\
\end{tabular} & 1.225 & 0.004176 & 0.072 & 0.002409 \\
\hline & CL02-578 & 1 & 1.185 & 1.323 & 0.0694 & 11.80 & & & & & & & & \begin{tabular}{|l|}
0.014918 \\
0.0149
\end{tabular} & & & & & & \\
\hline & & & 1.189 & 1.330 & 0.0708 & 12.00 & & & 1527 & & & & & \begin{tabular}{|l|}
0.015558 \\
\end{tabular} & & & & & & \\
\hline & & 3 & $\begin{array}{l}1.173 \\
1.775\end{array}$ & $\begin{array}{l}1.314 \\
1315\end{array}$ & 0.0705 & $\begin{array}{l}12.49 \\
1.29\end{array}$ & 0.31 & 0.30 & $\begin{array}{l}1601 \\
1592\end{array}$ & 34 & 33 & 1570 & & \begin{tabular}{|l|}
0.015247 \\
0015104
\end{tabular} & \begin{tabular}{|l|l|}
0.015207 \\
\end{tabular} & \begin{tabular}{|l|}
0.000264984 \\
\end{tabular} & 1.180 & 0.007388 & 0.070 & 0.000561 \\
\hline & & 4 & 1.175 & 1.315 & 0.0701 & 12.29 & & & 1592 & & 28 & & & \begin{tabular}{|l|l|}
0.015104 \\
\end{tabular} & & & & & & \\
\hline & Air & 1 & $\begin{array}{ll}1.174 \\
1160\end{array}$ & $\begin{array}{l}1.313 \\
1309\end{array}$ & 0.0695 & $\begin{array}{l}12.9 \\
12 .\end{array}$ & & & $\begin{array}{l}1705 \\
1051\end{array}$ & & & & & \begin{tabular}{|l|l|}
0.014821 \\
\end{tabular} & & & & & & \\
\hline & & $\frac{2}{3}$ & $\begin{array}{l}1.169 \\
1.167\end{array}$ & $\begin{array}{l}.308 \\
1.307 \\
\end{array}$ & $\begin{array}{l}0.0698 \\
0.0969\end{array}$ & $\begin{array}{l}12.6 \\
12.5 \\
\end{array}$ & 0.54 & 0.53 & $\begin{array}{l}1651 \\
1630 \\
\end{array}$ & 75 & 73.58 & 1696 & & \begin{tabular}{|l|}
0.014877 \\
0.014926 \\
\end{tabular} & 4885 & 4 467679E-05 & & & & 0000178 \\
\hline & & $\frac{3}{4}$ & $\frac{1.161}{1.175}$ & $\frac{1.307}{1.315}$ & $\begin{array}{l}0.06999 \\
0.0697 \\
\end{array}$ & $\begin{array}{l}12.5 \\
13.7 \\
\end{array}$ & 0.54 & 0.53 & 1030 & & 73.58 & 1696 & & \begin{tabular}{|l|}
0.014926 \\
0.014916 \\
\end{tabular} & 0.014885 & \begin{tabular}{|l}
$4.67679 \mathrm{E}-05$ \\
\end{tabular} & 1.171 & 0.004006 & 0.070 & 0.000178 \\
\hline \multirow[t]{13}{*}{ N1059 } & CL02-576 & & 1.243 & 1.386 & 0.0713 & 16.59 & & & 2078 & & & & & \begin{tabular}{|l|}
0.016491 \\
\end{tabular} & & & & & & \\
\hline & & $\frac{2}{3}$ & 1.238 & $\begin{array}{l}1.383 \\
1.295 \\
\end{array}$ & 0.0730 & 17.36 & & & 2074 & & & & & 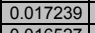 & & & & & & \\
\hline & - & 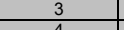 & $\frac{1.242}{1248}$ & $\begin{array}{l}1.385 \\
1205 \\
\end{array}$ & $\begin{array}{ll}0.0714 \\
0.0725\end{array}$ & $\begin{array}{l}17.66 \\
12.16\end{array}$ & 2.56 & 2.51 & $\begin{array}{l}2206 \\
1135\end{array}$ & 347 & 341 & 1948 & & $\begin{array}{l}0.016527 \\
0017909 \\
\end{array}$ & \begin{tabular}{|l|l|}
0.016964 \\
\end{tabular} & 0.000534857 & 1.243 & 0.004292 & 0.072 & 0.001079 \\
\hline & CL02-577 & $\frac{4}{1}$ & $\begin{array}{l}1.248 \\
1282 \\
\end{array}$ & $\begin{array}{l}1.395 \\
1429 \\
\end{array}$ & \begin{tabular}{|l|}
0.0735 \\
0
\end{tabular} & $\frac{12.16}{934}$ & - & - & $\frac{1435}{1106}$ & - & - & & $\begin{array}{l}-1.48 \\
-139 \\
\end{array}$ & 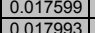 & & & & & & \\
\hline & & $\frac{2}{2}$ & $\begin{array}{l}1.283 \\
1.287\end{array}$ & $\begin{array}{l}1.423 \\
1.424\end{array}$ & \begin{tabular}{|l|}
0.0703 \\
\end{tabular} & $\begin{array}{l}12.79 \\
12.79\end{array}$ & & & $\begin{array}{l}1649 \\
1649\end{array}$ & & & & 1.00 & \begin{tabular}{|l|}
0.016494 \\
\end{tabular} & & & & & & \\
\hline & 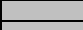 & 3 & 1.276 & 1.421 & 0.0726 & 12.10 & 1.52 & 1.49 & 1464 & 227 & 223 & 1421 & & \begin{tabular}{|l|l|}
0.017509 \\
\end{tabular} & \begin{tabular}{|l|}
0.01733 \\
\end{tabular} & \begin{tabular}{|l|}
0.000521499 \\
\end{tabular} & 1.279 & 0.004061 & 0.072 & 0.001266 \\
\hline & CL02-578 & $\frac{4}{1}$ & 1.275 & 1.419 & 0.0722 & 11.99 & & & 1465 & & & & & \begin{tabular}{|l|l|}
0.017325 \\
\end{tabular} & & & & & & \\
\hline & CLLOZ-5/8 & & $\begin{array}{l}1.232 \\
1.242 \\
\end{array}$ & $\begin{array}{l}1.369 \\
1.382 \\
\end{array}$ & \begin{tabular}{|l|}
0.0688 \\
0.0702 \\
\end{tabular} & 16.84 & & & 2179 & 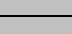 & $-5+2+$ & & & \begin{tabular}{|l|}
0.015934 \\
\end{tabular} & & & 1.240 & 0.014909 & 0.071 & 0.001239 \\
\hline & & & 1.241 & 1.384 & \begin{tabular}{|l|}
0.0715 \\
\end{tabular} & 18. & 1.75 & 1.71 & 2260 & 237 & 232 & 2084 & & \begin{tabular}{|l|}
0.016567 \\
\end{tabular} & 0.016388 & \begin{tabular}{|l}
0.000382348 \\
\end{tabular} & & & & 0.001200 \\
\hline & & 4 & 1.238 & 1.382 & \begin{tabular}{|l|}
0.0718 \\
\end{tabular} & 14.69 & & & 1815 & & & & & \begin{tabular}{|l|l|}
0.016664 \\
\end{tabular} & & & & & & \\
\hline & Air & $\frac{1}{2}$ & $\begin{array}{l}1.176 \\
1168\end{array}$ & \begin{tabular}{|l|l|}
1.311 \\
1.303
\end{tabular} & \begin{tabular}{|l|}
0.0677 \\
0.073 \\
\end{tabular} & $\begin{array}{l}19.5 \\
17.4\end{array}$ & 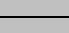 & & 2710 & & & & & 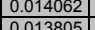 & & & & & & \\
\hline & & $\frac{2}{3}$ & $\begin{array}{l}\frac{1.168}{1.171} \\
171\end{array}$ & $\begin{array}{l}1.303 \\
1.312 \\
\end{array}$ & $\begin{array}{l}0.0673 \\
0.0703 \\
\end{array}$ & 14.5 & 2.05 & 2.01 & $\frac{2444}{1872}$ & 374 & 366.57 & 2276 & & \begin{tabular}{|l|}
0.013805 \\
0.015156 \\
\end{tabular} & 0.014629 & 0.000804831 & 1.171 & 0.003557 & 0.069 & 0.001886 \\
\hline & & 4 & 1.168 & 1.310 & $\begin{array}{ll}0.0712 \\
\end{array}$ & 16.5 & & & 2077 & & & & & \begin{tabular}{|l|}
0.015492 \\
\end{tabular} & & & & & & \\
\hline \multirow[t]{13}{*}{ V747 } & CL02-576 & $\frac{1}{2}$ & 0.863 & 1.001 & 0.0693 & 13. & & & 1845 & & & & & \begin{tabular}{|l|l|}
0.011044 \\
\end{tabular} & & & & & & \\
\hline & & $\frac{2}{3}$ & $\begin{array}{l}0.874 \\
0.868\end{array}$ & \begin{tabular}{l|l|}
1.008 \\
1.008
\end{tabular} & $\begin{array}{ll}0.0669 \\
0.0702\end{array}$ & $\begin{array}{l}13.73 \\
14.51\end{array}$ & 0.36 & 0.35 & $\begin{array}{l}1953 \\
1877 \\
\end{array}$ & 316 & 310 & 1854 & & & 0.011109 & \begin{tabular}{|c|}
000517376 \\
\end{tabular} & 0.867 & 0.005321 & 0.069 & 0.001714 \\
\hline & & 3 & $\begin{array}{l}0.868 \\
0.863 \\
\end{array}$ & $\begin{array}{l}1.008 \\
1.005 \\
\end{array}$ & 0.07 & $\begin{array}{l}1.51 \\
13.77 \\
\end{array}$ & & & 1742 & & & & & $\mid$\begin{tabular}{|l}
0.011394 \\
0.011603 \\
\end{tabular} & & & 0.000 & 0.000321 & 0.000 & $0.001 / 14$ \\
\hline & CL02-577 & 1 & 0.898 & 1.0 & 0.07 & 9.8 & & & 1222 & & & & & \begin{tabular}{|l|}
0.012321 \\
\end{tabular} & & & & & & \\
\hline & & 2 & 0.893 & 1.0 & 0.07 & 9.4 & & & 1223 & & & & & \begin{tabular}{|l|l|}
0.011643 \\
\end{tabular} & & & & & & \\
\hline & & 3 & 0.900 & 1.041 & 0.0707 & 9.66 & 0.24 & 0.23 & 1232 & 6 & 6 & 1226 & & 0.011955 & \begin{tabular}{|l|}
0.011973 \\
\end{tabular} & \begin{tabular}{|l|}
0.000384081 \\
\end{tabular} & 0.897 & 0.003164 & 0.071 & 0.000727 \\
\hline & & 4 & 0.900 & 1.6 & 0.0705 & & & & & & & & & & & & & & & \\
\hline & CL02-578 & & 0. & & 0.6 & 15. & & & 1789 & & & & & 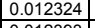 & & & & & & \\
\hline & & 2 & $\begin{array}{l}0.854 \\
0.875 \\
\end{array}$ & $\begin{array}{l}1.001 \\
1.011 \\
\end{array}$ & \begin{tabular}{l|l}
0.0736 \\
0.0684
\end{tabular} & $\frac{14.32}{13.76}$ & 0.56 & 0.55 & $\begin{array}{l}\frac{1685}{1871} \\
871\end{array}$ & 83 & 81 & 1798 & & \begin{tabular}{|l|}
0.010901 \\
\end{tabular} & \begin{tabular}{|l|l|l|l|}
0.01169 \\
\end{tabular} & \begin{tabular}{|l|}
0.00076343 \\
\end{tabular} & 0.865 & 0.009136 & 0.071 & 0.002566 \\
\hline & & 4 & 0.871 & 1.010 & 0.06 & 13. & & & 1848 & & & & & \begin{tabular}{|l|}
0.011141 \\
0.0114
\end{tabular} & & & & & & 0.002000 \\
\hline & Air & 1 & 0.865 & 1.0 & 0.0 & 15 & & & 19 & & & & & & & & & & & \\
\hline & & & $\begin{array}{l}0.866 \\
0.861\end{array}$ & 1.0 & 0.0 & $\frac{14}{11}$ & & & & & & & & & & & & & & \\
\hline & & 3 & 0.864 & 1.6 & 0.0 & 14 & 0.49 & 0.48 & 1886 & 77 & 75.75 & 1901 & & 0.0 & \begin{tabular}{|l|}
0.011242 \\
\end{tabular} & \begin{tabular}{|l|}
0.000144988 \\
\end{tabular} & 0.864 & 0.002365 & 0.070 & 0.000497 \\
\hline \multirow{14}{*}{ V884 } & CLL02-576 & 4 & \begin{tabular}{|l}
0.860 \\
0.986 \\
\end{tabular} & $\begin{array}{l}1.0 \\
1.12\end{array}$ & \begin{tabular}{|l|l|}
0.0 \\
0.0 \\
\end{tabular} & $\frac{14}{13}$ & & & \begin{tabular}{|l|}
1799 \\
1778
\end{tabular} & & & & & & & & & & & \\
\hline & & 2 & 0.981 & 1.1 .2 & \begin{tabular}{|l|l|}
0.065 \\
\end{tabular} & 13.6 & & & 1712 & & & & & \begin{tabular}{|l|}
0.012536 \\
0.01253 \\
\end{tabular} & & & & & & \\
\hline & & 3 & 0.95 & 1.1 & 0.06 & 13.4 & 0.19 & 0.18 & 17 & 37 & 36 & 1749 & & & 0.012571 & \begin{tabular}{|l|}
0.00021365 \\
\end{tabular} & 0.988 & 0.005334 & 0.069 & 0.000494 \\
\hline & & $\frac{4}{4}>3$ & 0.993 & 1.1 & 0.07 & 13. & & & 1723 & & & & & \begin{tabular}{|l|l|}
0.01285 \\
\end{tabular} & & & & & & \\
\hline & CL02-577 & 1 & 1.0 & 1. & 0.0 & 10 & & & 1266 & & & & & 83 & & & & & & \\
\hline & & $\frac{2}{3}$ & $\begin{array}{l}1.013 \\
1015 \\
\end{array}$ & & & & 037 & 036 & $\frac{125}{111}$ & 54 & 53 & 1215 & & & 0 013951 & 610001 & 1013 & 121 & 0072 & 0001471 \\
\hline & & $\frac{3}{4}$ & $\begin{array}{l}1.015 \\
1015 \\
\end{array}$ & $\begin{array}{l}1.164 \\
1.158 \\
\end{array}$ & 0.0743 & $\frac{10.10}{9.43}$ & 0.37 & 0.36 & $\frac{116}{111}$ & 54 & 53 & 1215 & & 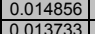 & 0.013951 & 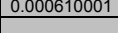 & 7.013 & 0.003427 & 0.012 & 0.001471 \\
\hline & CL02-578 & 1 & 0.95 & 1. & 0.0668 & 12.82 & & & 1833 & & & & & \begin{tabular}{|l|}
0.011634 \\
\end{tabular} & & & & & & \\
\hline & & $\frac{2}{2}$ & 0.987 & 1.1. & 0.0 & & & & & & & & & & & & & & & \\
\hline & & 3 & 0.988 & 1. & \begin{tabular}{|l|l|}
0.06 \\
\end{tabular} & 13 & 0.30 & 0.29 & 1848 & 62.59 & 61 & 1805 & & 0.0 & 0.012116 & 0.000734811 & 0.989 & 0.001911 & 0.069 & 0.001671 \\
\hline & & 4 & 0.9 & 1. & 0.0 & 13 & & & 17 & & & & & & & & & & & \\
\hline & Air & $\frac{1}{2}$ & & & 0.0 & 14 & & & $\frac{19}{16}$ & & & & & & & & & & & \\
\hline & & $\frac{2}{3}$ & 0.989 & $\frac{1.130}{1.124}$ & $\begin{array}{ll}0.0704 \\
0.0696\end{array}$ & 13. & 0.65 & 0.64 & $\begin{array}{l}1676 \\
1884\end{array}$ & 119 & 116.47 & 1818 & & & 0.012693 & 0.000279043 & 0.989 & 0.003021 & 0.070 & 0.000704 \\
\hline & & 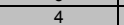 & 0.992 & 1.132 & 0.0701 & 13.7 & & & 1768 & & & & & & & & & & & \\
\hline
\end{tabular}




\begin{tabular}{|c|c|c|c|c|c|c|c|}
\hline 0 -ring & Condition & $\begin{array}{l}\text { Average } \\
\text { Break Load } \\
\text { (Ibs) }\end{array}$ & $\begin{array}{l}\text { Std. Dev. } \\
\text { Break Load } \\
\text { (lbs) }\end{array}$ & $\begin{array}{l}95 \% \text { Conf. Int. } \\
\text { Break Load } \\
\text { (lbs) }\end{array}$ & $\begin{array}{l}\text { Average } \\
\text { Break Stress } \\
\text { (psi) }\end{array}$ & $\begin{array}{l}\text { Std. Dev. } \\
\text { Break Stress } \\
\text { (psi) }\end{array}$ & $\begin{array}{l}\text { 95\% Conf. Int. } \\
\text { Break Stress } \\
\text { (psi) }\end{array}$ \\
\hline \multirow[t]{4}{*}{ N1059 } & CL02-576 & 15.94 & 2.56 & 2.51 & 1948 & 347 & 341 \\
\hline & CL02-577 & 11.56 & 1.52 & 1.49 & 1421 & 227 & 223 \\
\hline & CL02-578 & 16.56 & 1.75 & 1.71 & 2084 & 237 & 232 \\
\hline & Air & 16.98 & 2.05 & 2.01 & 2276 & 374 & 367 \\
\hline \multirow[t]{4}{*}{ N674 } & CL02-576 & 15.71 & 1.26 & 1.24 & 1803 & 140 & 138 \\
\hline & CL02-577 & 12.08 & 1.14 & 1.11 & 1255 & 116 & 113 \\
\hline & CL02-578 & 15.33 & 2.58 & 2.52 & 1811 & 28 & 28 \\
\hline & Air & 19.32 & 0.11 & 0.11 & 2495 & 43 & 42 \\
\hline \multirow[t]{4}{*}{ NO497 } & CL02-576 & 12.34 & 0.12 & 0.12 & 1598 & 35 & 34 \\
\hline & CL02-577 & 11.22 & 0.16 & 0.16 & 1392 & 115 & 113 \\
\hline & CL02-578 & 12.15 & 0.31 & 0.30 & 1570 & 34 & 33 \\
\hline & Air & 12.95 & 0.54 & 0.53 & 1696 & 75 & 74 \\
\hline \multirow[t]{4}{*}{ V747 } & CL02-576 & 13.98 & 0.36 & 0.35 & 1854 & 316 & 310 \\
\hline & CL02-577 & 9.65 & 0.24 & 0.23 & 1226 & 6 & 6 \\
\hline & CL02-578 & 14.26 & 0.56 & 0.55 & 1798 & 83 & 81 \\
\hline & Air & 14.56 & 0.49 & 0.48 & 1901 & 77 & 76 \\
\hline \multirow[t]{4}{*}{ V884 } & CL02-576 & 13.23 & 0.19 & 0.18 & 1749 & 37 & 36 \\
\hline & CL02-577 & 9.94 & 0.37 & 0.36 & 1215 & 54 & 53 \\
\hline & CL02-578 & 13.14 & 0.30 & 0.29 & 1805 & 63 & 61 \\
\hline & Air & 13.8575 & 0.65 & 0.64 & 1818 & 119 & 116 \\
\hline
\end{tabular}

\begin{tabular}{|c|c|c|c|c|c|c|c|c|c|c|c|}
\hline 0-ring & Exposure & $\begin{array}{l}\text { Average } \\
\text { Inside } \\
\text { Diameter } \\
\text { (in) }\end{array}$ & $\begin{array}{l}95 \% \text { Conf. Int. } \\
\text { Inside } \\
\text { Diameter } \\
\text { (in) }\end{array}$ & $\begin{array}{l}\text { Average } \\
\text { O-ring } \\
\text { Thickness } \\
\text { (in) }\end{array}$ & $\begin{array}{l}95 \% \text { Conf. Int. } \\
\text { O-ring } \\
\text { Thickness } \\
\text { (in) }\end{array}$ & $\begin{array}{c}\text { Average } \\
\text { Break Load } \\
\text { (Ibs) }\end{array}$ & $\begin{array}{l}\text { 95\% Conf. Int. } \\
\text { Break Load } \\
\text { (Ibs) }\end{array}$ & $\begin{array}{c}\text { Average } \\
\text { Break Stress } \\
\text { (psi) }\end{array}$ & $\begin{array}{l}\text { 95\% Conf. Int. } \\
\text { Break Stress } \\
\text { (psi) }\end{array}$ & $\begin{array}{c}\text { Average } \\
\text { Volume } \\
\text { (in^3) }\end{array}$ & $\begin{array}{c}95 \% \text { C.I. } \\
\text { Volume } \\
\text { (in^3) }\end{array}$ \\
\hline \multirow[t]{4}{*}{ N1059 } & CL02-576 & 1.243 & 0.004292 & 0.072 & 0.001079 & 15.94 & 2.51 & 1948.3 & 340.5 & 0.016964 & 0.000535 \\
\hline & CL02-577 & 1.279 & 0.004061 & 0.072 & 0.001266 & 11.56 & 1.49 & 1421.0 & 222.6 & 0.017330 & 0.000521 \\
\hline & CL02-578 & 1.240 & 0.014909 & 0.071 & 0.001239 & 16.56 & \begin{tabular}{ll|}
1.71 \\
\end{tabular} & 2084.4 & 232.4 & 0.016388 & 0.000382 \\
\hline & Air & 1.171 & 0.003557 & 0.069 & 0.001886 & 16.98 & 2.01 & 2275.7 & 366.6 & 0.014629 & 0.000805 \\
\hline \multirow[t]{4}{*}{ N674 } & CL02-576 & 1.033 & 0.001661 & 0.074 & 0.000164 & 15.71 & 1.24 & 1803.4 & 137.6 & 0.015169 & 0.000078 \\
\hline & CL02-577 & 1.071 & 0.003092 & 0.078 & 0.000709 & 12.08 & 1.11 & 1255.4 & 113.3 & 0.017373 & 0.000306 \\
\hline & CL02-578 & 1.030 & 0.000702 & 0.073 & 0.000860 & 15.33 & 2.52 & 1810.6 & 27.7 & 0.014671 & 0.000345 \\
\hline & Air & 0.990 & 0.000775 & 0.070 & 0.000476 & 19.32 & 0.11 & 2494.8 & 42.0 & 0.012905 & 0.000178 \\
\hline \multirow[t]{4}{*}{ NO497 } & CL02-576 & 1.184 & 0.003321 & 0.070 & 0.000445 & 12.34 & 0.12 & 1598.4 & 34.4 & 0.015211 & 0.000186 \\
\hline & CL02-577 & 1.225 & 0.004176 & 0.072 & 0.002409 & 11.22 & 0.16 & 1392.0 & 112.8 & 0.016466 & 0.001098 \\
\hline & CL02-578 & 1.180 & 0.007388 & 0.070 & 0.000561 & 12.15 & 0.30 & 1569.8 & 33.2 & 0.015207 & 0.000265 \\
\hline & Air & 1.171 & 0.004006 & 0.070 & 0.000178 & 12.95 & 0.53 & 1696.4 & 73.6 & 0.014885 & 0.000047 \\
\hline \multirow[t]{4}{*}{ V747 } & CL02-576 & 0.867 & 0.005321 & 0.069 & 0.001714 & 13.98 & 0.35 & 1854.4 & 309.6 & 0.011109 & 0.000517 \\
\hline & CL02-577 & 0.897 & 0.003164 & 0.071 & 0.000727 & 9.65 & 0.23 & 1225.5 & 5.6 & 0.011973 & 0.000384 \\
\hline & CL02-578 & 0.865 & 0.009136 & 0.071 & 0.002566 & 14.26 & 0.55 & 1798.3 & 81.3 & 0.011690 & 0.000763 \\
\hline & Air & 0.864 & 0.002365 & 0.070 & 0.000497 & 14.56 & 0.48 & 1901.4 & 75.8 & 0.011242 & 0.000145 \\
\hline \multirow[t]{4}{*}{ V884 } & CL02-576 & 0.988 & 0.005334 & 0.069 & 0.000494 & 13.23 & 0.18 & 1749.2 & 36.4 & 0.012571 & 0.000214 \\
\hline & CL02-577 & 1.013 & 0.003421 & 0.072 & 0.001471 & 9.94 & 0.36 & 1215.2 & 53.2 & 0.013951 & 0.000610 \\
\hline & CL02-578 & 0.989 & 0.001911 & 0.068 & 0.001671 & 13.14 & 0.29 & 1804.7 & 61.3 & 0.012116 & 0.000735 \\
\hline & Air & 0.989 & 0.003021 & 0.070 & 0.000704 & 13.86 & 0.64 & 1817.7 & 116.5 & 0.012693 & 0.000279 \\
\hline
\end{tabular}




\section{Appendix C: \\ Bar Graphs with 95\% Confidence Bands}




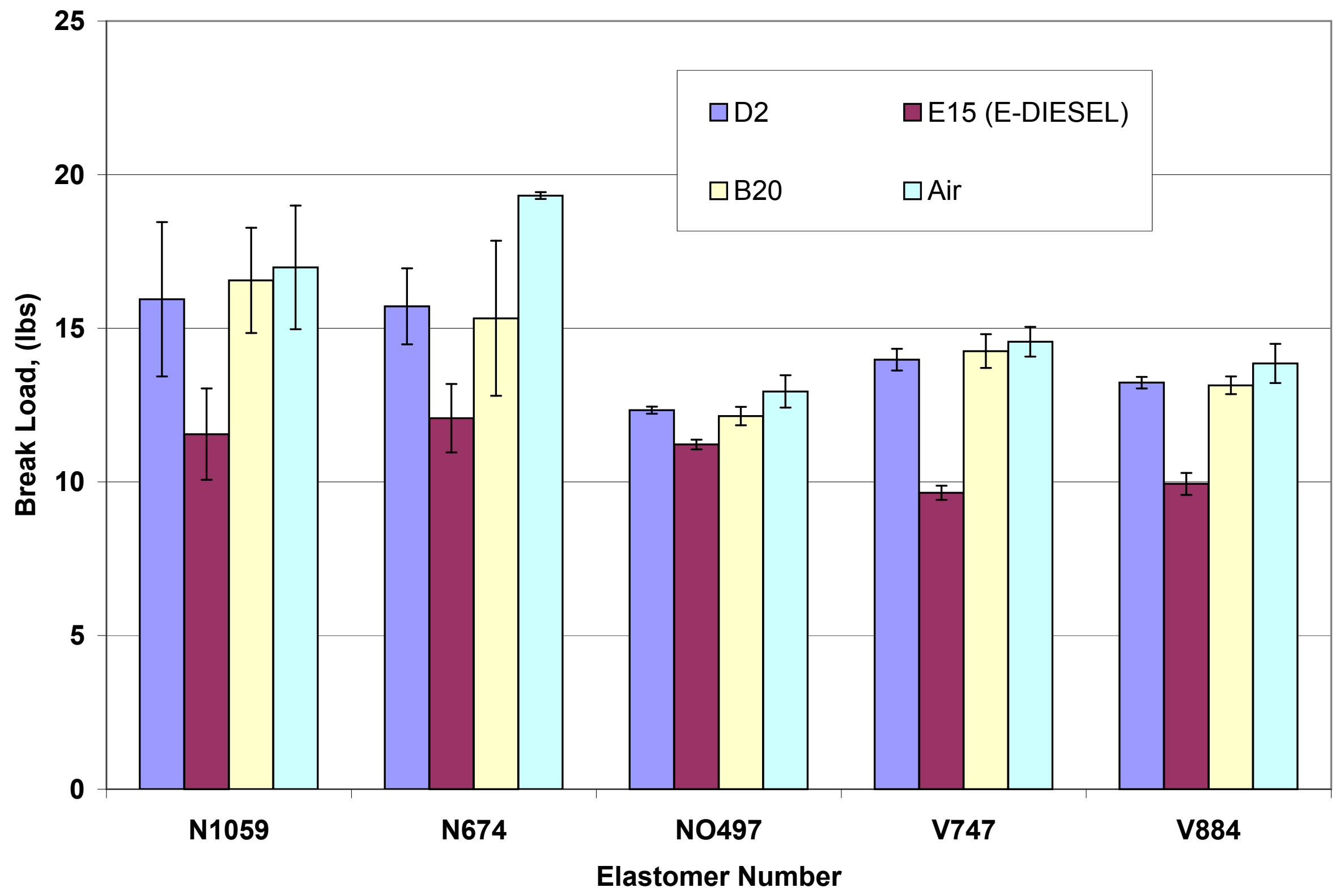




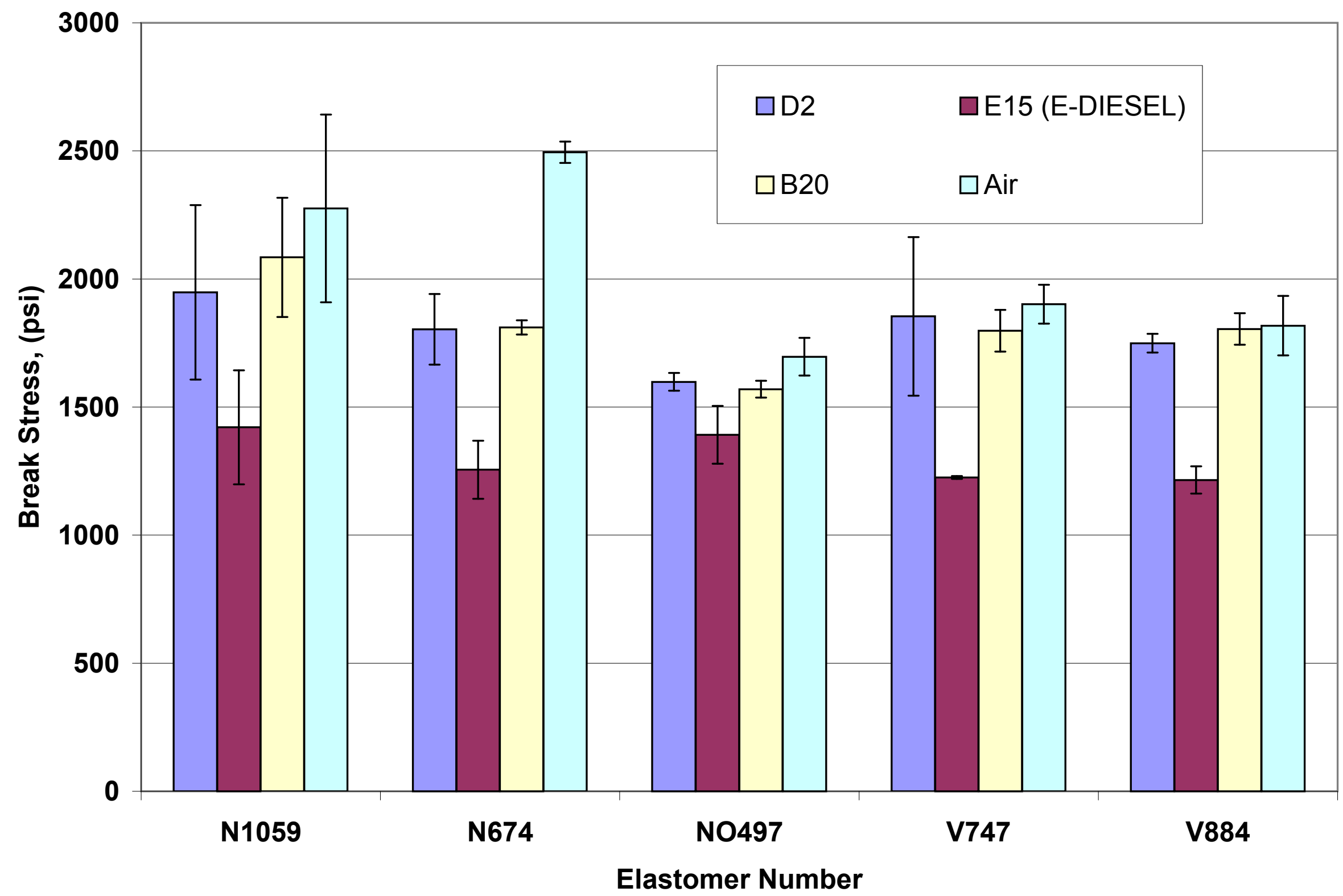




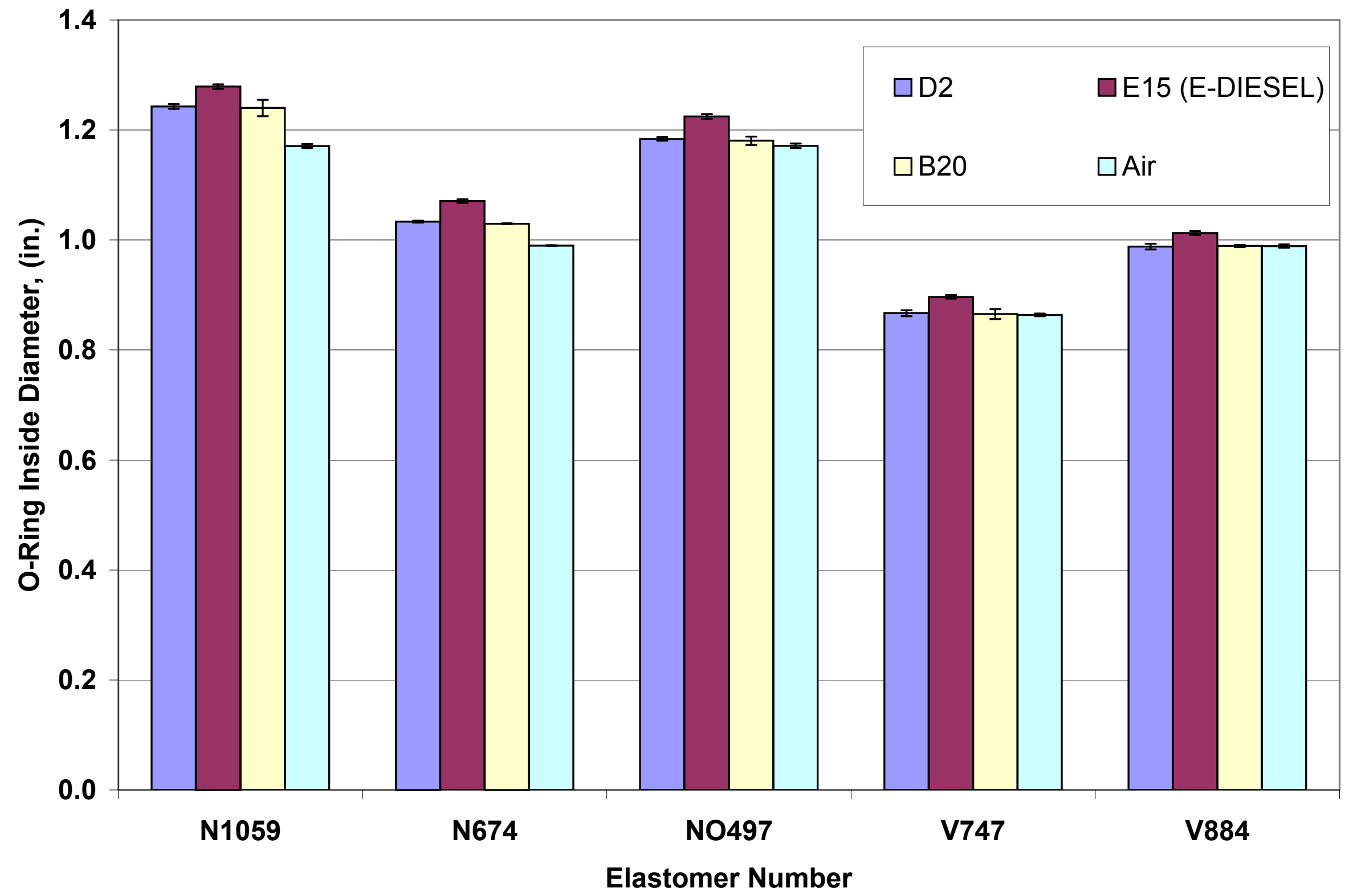




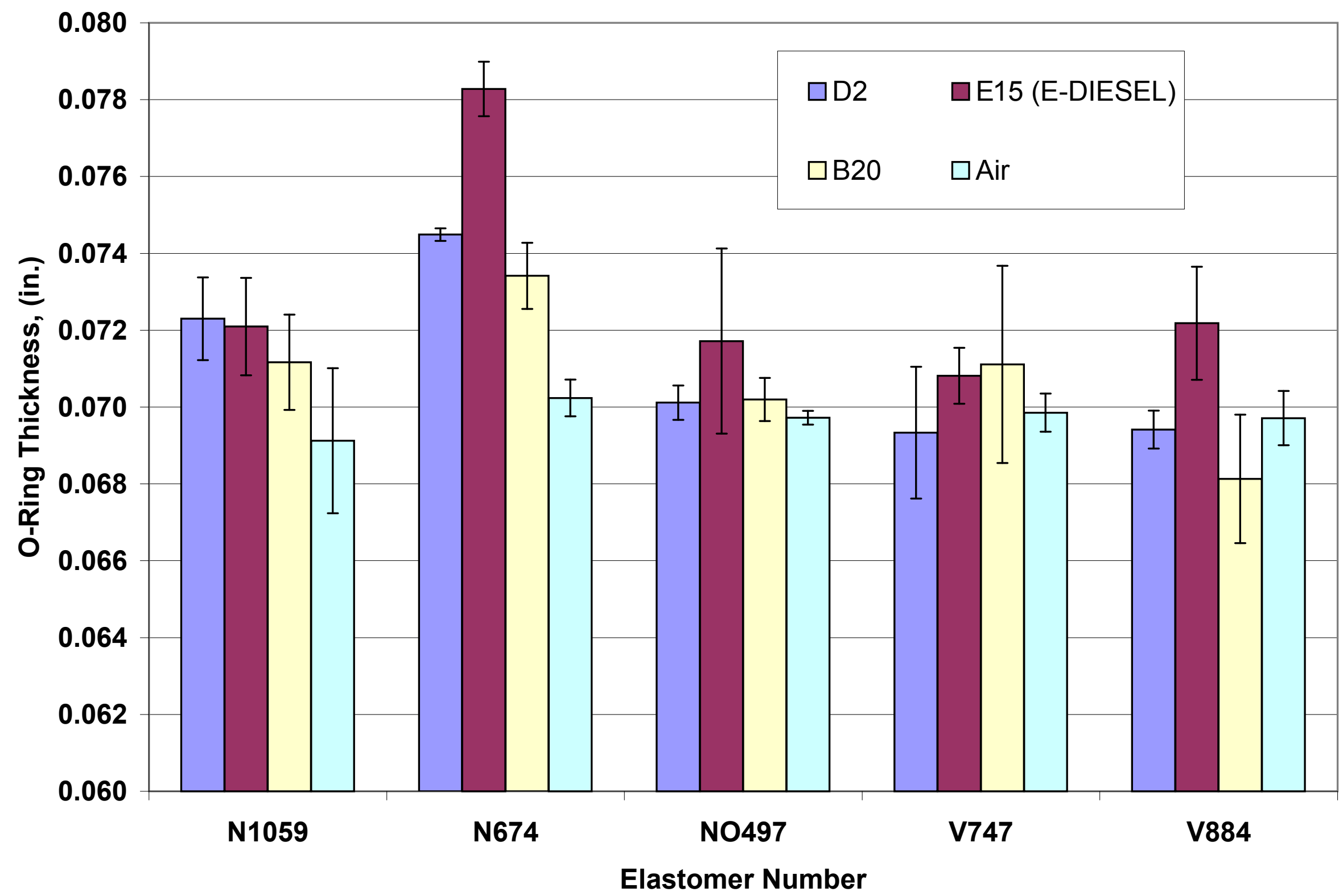




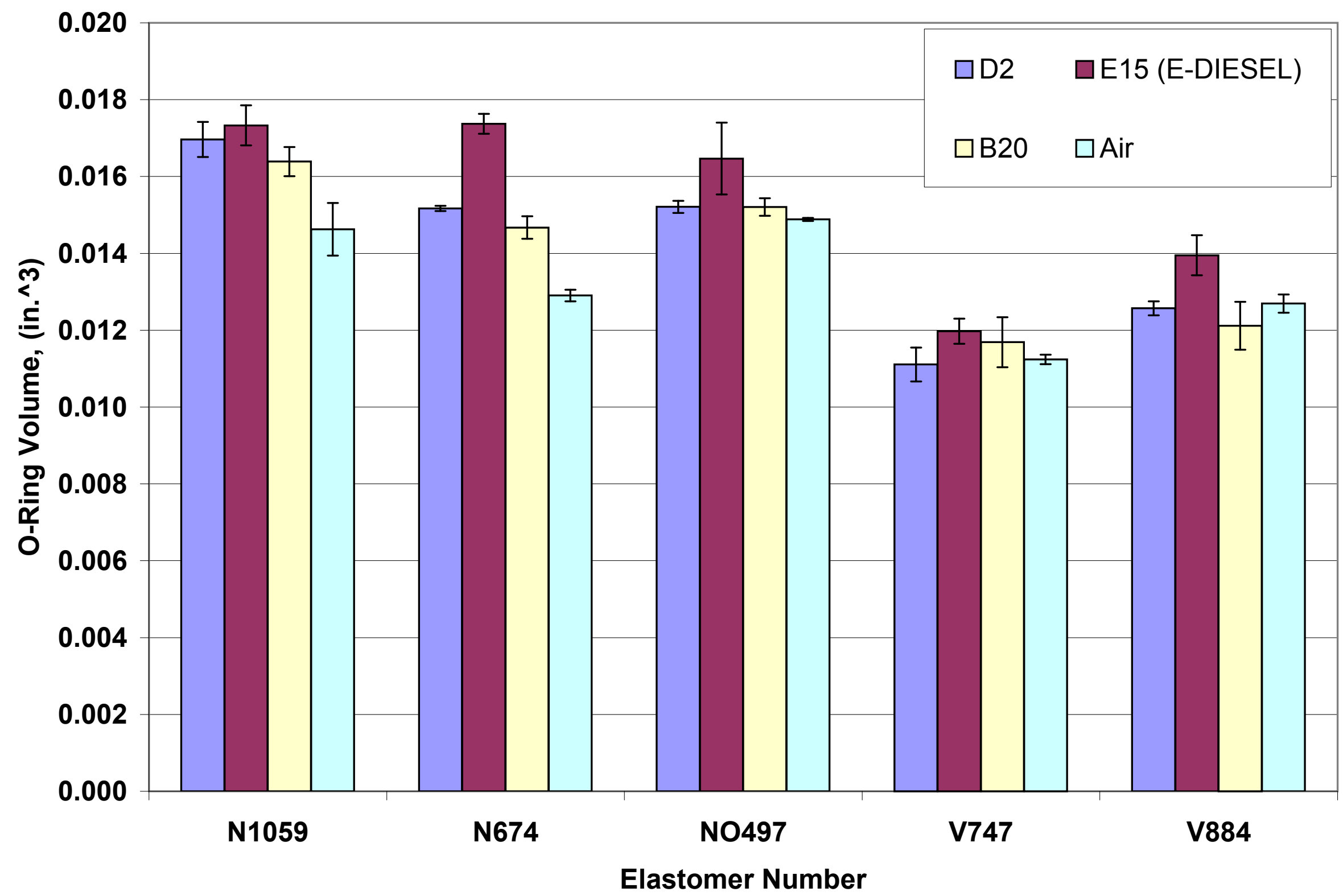




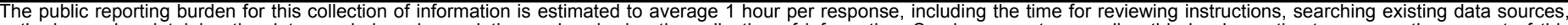

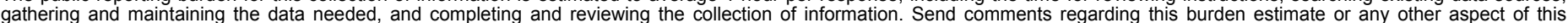

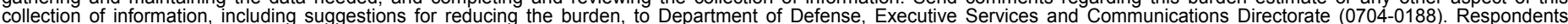

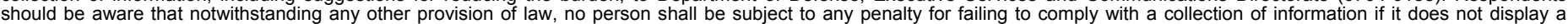

should be awa

PLEASE DO NOT RETURN YOUR FORM TO THE ABOVE ORGANIZATION.

\begin{tabular}{l|l|l|l} 
1. REPORT DATE $(D D-M M-Y Y Y Y)$ & 2. & REPORT TYPE & 3. DATES COVERED (FrOm - TO)
\end{tabular}

November 2005

Technical Report

4. TITLE AND SUBTITLE

Elastomer Compatibility Testing of Renewable Diesel Fuels 5a. CONTRACT NUMBER

DE-AC36-99-GO10337

5b. GRANT NUMBER

5c. PROGRAM ELEMENT NUMBER

5d. PROJECT NUMBER

NREL/TP-540-38834

5e. TASK NUMBER

FC06.9400

R.L. McCormick

National Renewable Energy Laboratory

5f. WORK UNIT NUMBER
7. PERFORMING ORGANIZATION NAME(S) AND ADDRESS(ES)

National Renewable Energy Laboratory

1617 Cole Blvd.

Golden, CO 80401-3393

9. SPONSORING/MONITORING AGENCY NAME(S) AND ADDRESS(ES)
8. PERFORMING ORGANIZATION REPORT NUMBER

NREL/TP-540-38834
10. SPONSOR/MONITOR'S ACRONYM(S) NREL

11. SPONSORING/MONITORING AGENCY REPORT NUMBER

12. DISTRIBUTION AVAILABILITY STATEMENT

National Technical Information Service

U.S. Department of Commerce

5285 Port Royal Road

Springfield, VA 22161

13. SUPPLEMENTARY NOTES

14. ABSTRACT (Maximum 200 Words)

In this study, the integrity and performance of six elastomers were tested with ethanol-diesel and biodiesel fuel

blends.

\section{SUBJECT TERMS}

elastomers; ethanol-diesel; biodiesel; renewable diesel; fuel blends; elastomer compability

\begin{tabular}{|c|c|c|}
\hline $\begin{array}{l}\text { a. REPORT } \\
\text { Unclassified }\end{array}$ & $\begin{array}{l}\text { b. ABSTRACT } \\
\text { Unclassified }\end{array}$ & $\begin{array}{l}\text { c. THIS PAGE } \\
\text { Unclassified }\end{array}$ \\
\hline
\end{tabular}

\begin{tabular}{l|l|} 
17. LIMITATION \\
OF ABSTRACT \\
UL
\end{tabular}

19a. NAME OF RESPONSIBLE PERSON

19b. TELEPHONE NUMBER (Include area code) 